\title{
Electrocardiographic changes following a cardiac stab wound. Case report and review of the literature
}

L. M. A. SHAW
M.B. B.S.
A. LAHIRI

M.B. B.S.

\author{
M. W. Millar-Craig \\ M.D., M.R.C.P. \\ Department of Cardiology, Northwick Park Hospital and \\ Clinical Research Centre, Harrow, Middlesex
}

\begin{abstract}
Summary
A patient is described who sustained a cardiac stab wound which was associated with ECG evidence of a full thickness myocardial infarction. Subsequent investigation showed a localized area of dyskinesia on left ventricular angiography, but normal thallium myocardial perfusion and normal anatomy on coronary angiography. The patient was managed conservatively and made a complete and uneventful recovery. It is concluded that extensive ECG changes following a cardiac stab wound need not be associated with major coronary artery damage.
\end{abstract}

\section{Introduction}

Thoracic stab wounds which involve the heart may cause injury to the myocardium, coronary arteries, valve structures or the great vessels. In the past there has been considerable interest in the management of these injuries (De Wet Lubbe, Janson and Barnard, 1975; Espada et al., 1975). Electrocardiography would normally be regarded as a basic initial investigation in such a case. A case is described in which electrocardiographic changes of an acute full thickness anterior myocardial infarction occurred in the absence of any significant coronary artery damage.

\section{Case report}

A previously fit 18-year-old man sustained 3 knife wounds in a fight. These wounds were in the 4th intercostal space at the left sternal edge, in the 7 th intercostal space at the right anterior axillary line and subcostally at the right mid-axillary line. On arrival at hospital he was unconscious, pale and shivering. His BP was $90 \mathrm{mmHg}$ systolic with a pulse rate of 110 beats $/ \mathrm{min}$. His jugular venous pressure was not raised and the heart sounds were clearly heard and were normal. After immediate resuscitation with saline followed by 2 units of blood over the ensuing $2 \mathrm{hr}$, his BP rose to $110 / 60 \mathrm{mmHg}$, his pulse rate dropped to 80 beats/min and he regained consciousness.

Radiography of the chest shortly after admission showed a normal cardiac size, but confirmed the presence of a left pneumothorax which was drained. Electrocardiography (ECG) $2 \mathrm{hr}$ after the stabbing, showed $\mathbf{Q}$ waves and loss of $\mathbf{R}$ waves in leads 1 , AVR, AVL, and V2 to 5; and peaked $T$ waves in leads V2, 3 and 4 (Fig. 1). Echocardiography showed no evidence of significant pericardial fluid.

The patient was managed conservatively in the Intensive Care Unit and, apart from a minor ches infection, made an uneventful recovery. Myocardia damage was confirmed by the presence of specific enzymatic rises (aspartate transaminase, 80 i.u./l; creatinine kinase, 808 i.u./l). Serial ECGs showed persistent $Q$ waves with return of the ST segment and $T$ waves to normal over the next 6 weeks. He continued to improve and was discharged with no medication on the 9th day after admission.

In view of his age and the persistent ECG changes suggestive of a full thickness anterior myocardial infarction, he was further investigated 4 months after the initial incident. At the time of investigation he was asymptomatic. A standard graded exercise test, performed on a treadmill using a Computer-Assisted System for Exercise (Marquette Electronics Ltd), showed a normal exercise tolerance, and ST segment variation remained within normal limits. ${ }^{201} \mathrm{Tl}-$ scintigraphy showed normal myocardial perfusion both at rest and during exercise. Left heart catheterization showed pressures in the left ventricle of $140 / 6$ $\mathrm{mmHg}$ with a left ventricular end diastolic pressure of $11 \mathrm{mmHg}$. Left ventricular angiography showed evidence of a localized area of dyskinesia, ventricular wall motion being otherwise normal (Fig. 2(a) and (b)). Selective coronary ciné-angiography showed entirely normal left and right coronary arteries (Fig. 3(a) and (b)). In particular there was no evidence of damage to the left anterior descending coronary artery (LAD). 

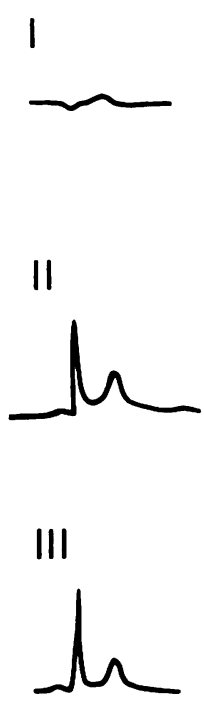

\section{AVR}

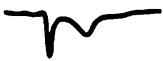

AVL

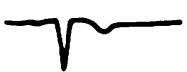

AVF

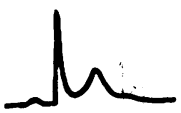

$V_{1}$

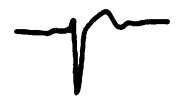

$V_{2}$
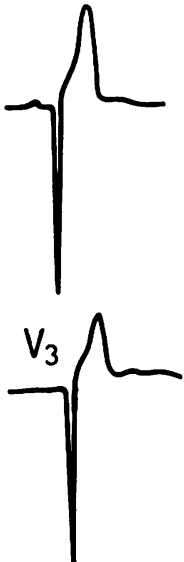

$\mathrm{V}_{4}$

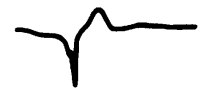

$V_{5}$

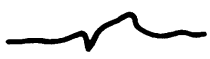

$V_{6}$

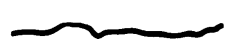

FIG. 1 The electrocardiogram recorded $2 \mathrm{hr}$ after the injury.
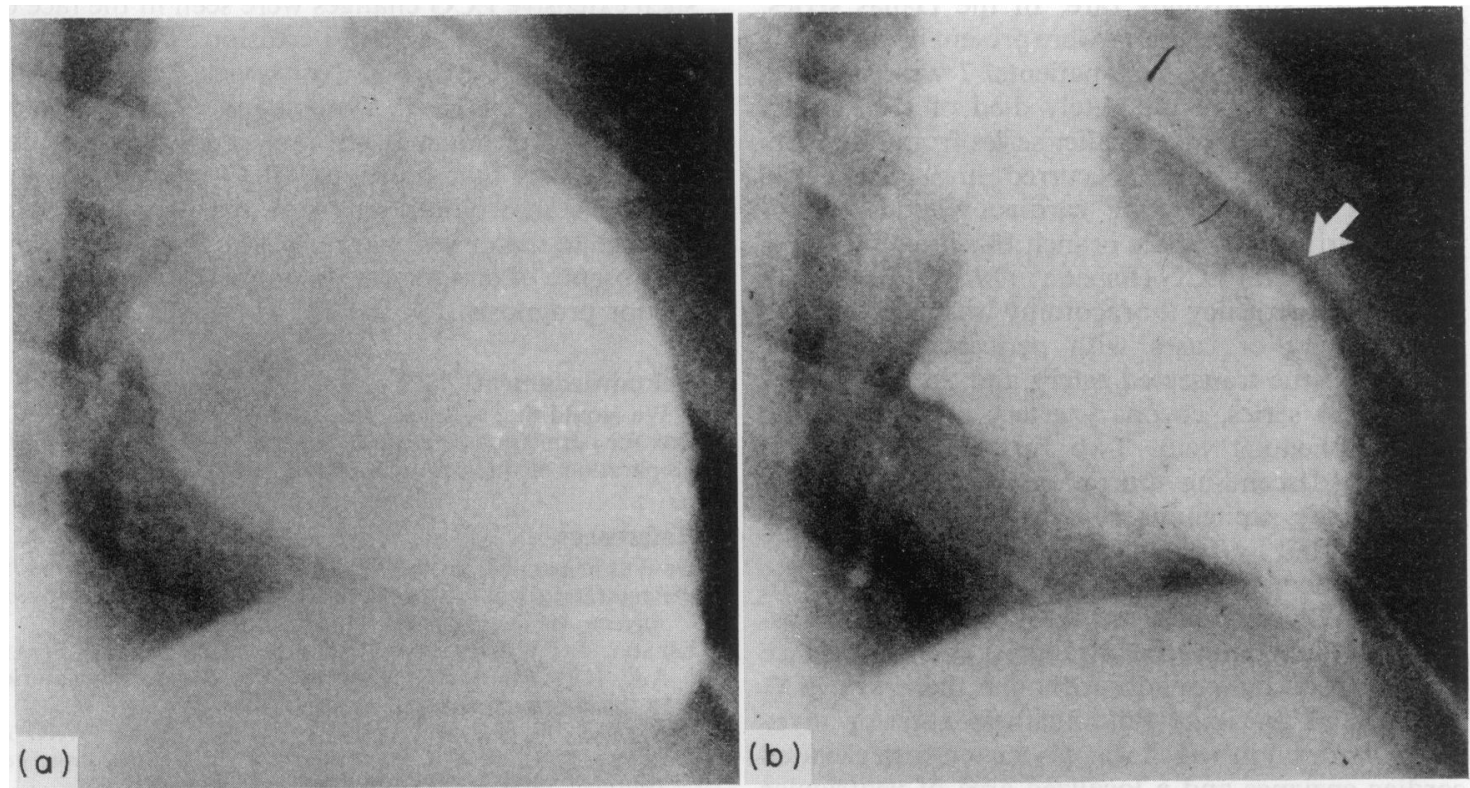

Fig. 2. Left ventricular ciné-angiogram (right anterior oblique view) 4 months after the injury. An end diastolic (a) and end systolic (b) frame is shown. The localized area of dyskinesia is indicated by the arrow. 

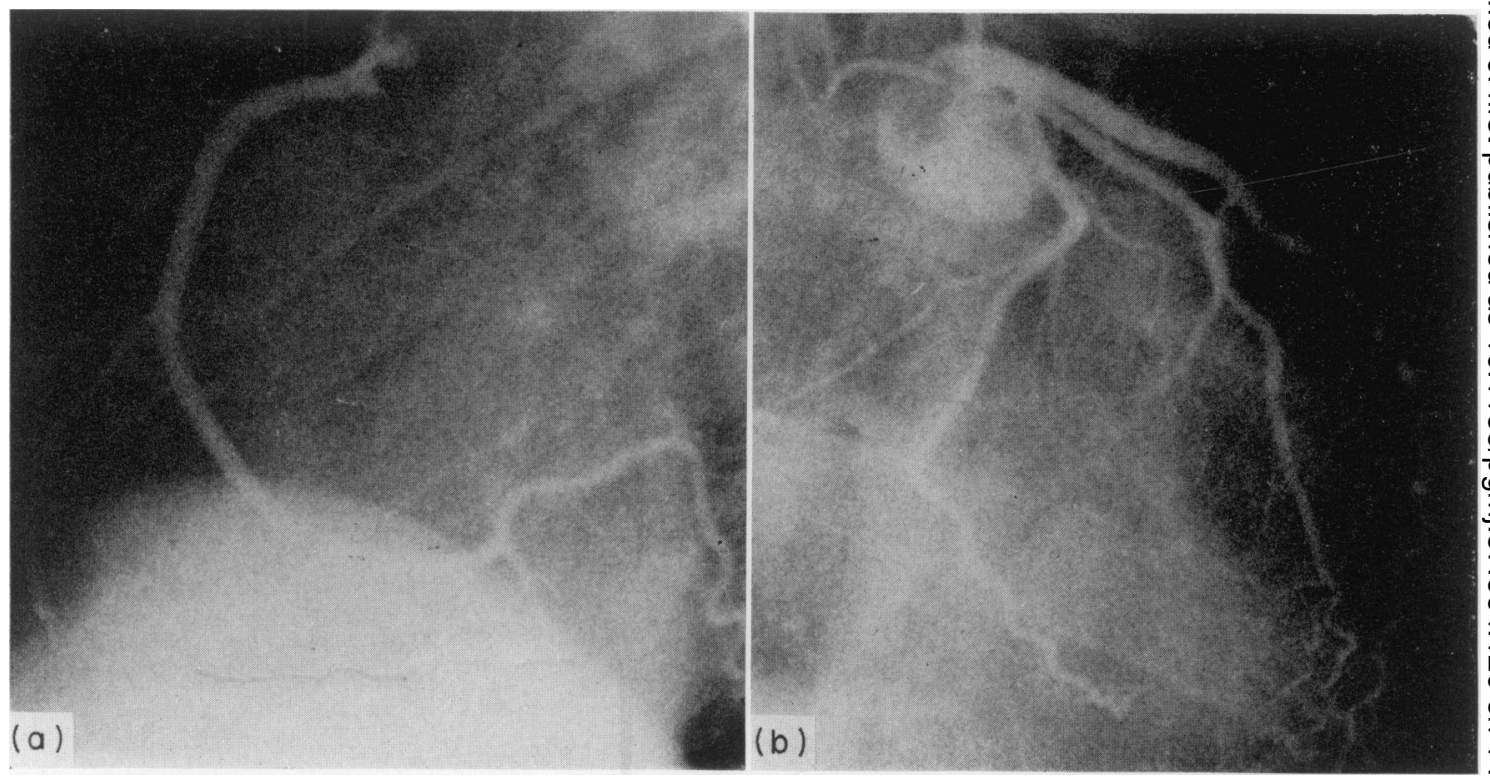

FIG. 3. Coronary angiograms obtained 4 months after the injury. Selective right coronary (left anterior oblique view) (a) and left coronary (right anterior oblique view) (b) injections. No abnormalities were present in either artery.

\section{Discussion}

Although penetrating injuries of the heart are not uncommon world-wide, injuries to the coronary arteries are surprisingly rare. In the Dallas series, coronary artery lacerations were present in only $4.4 \%$ of 500 cases. Of these 22 patients, 7 were dead on arrival and 5 more ultimately died of their injury (Rea et al., 1969). In a smaller series from Houston, coronary artery injury occurred in 9 out of 76 patients with penetrating cardiac wounds, all of whom had either bundle branch block or ST and T wave changes on ECG (Espada et al., 1975). In both series, an emergency thoracotomy was performed in the majority of cases with pericardial drainage, ligation of the transected artery and, in one case in the second series, coronary artery bypass grafting using saphenous vein. Two further cases of left anterior descending coronary artery laceration managed by saphenous vein bypass grafting have been reported by Tector et al. (1973).

This patient was treated conservatively because of the rapid clinical improvement following blood transfusion and pneumothorax drainage, and the absence of pericardial tamponade. Although there was ECG evidence of an acute full thickness anterior myocardial infarction which was associated with elevated cardiac enzymes and a localized area of ventricular dyskinesia on angiography, there was normal myocardial thallium perfusion and normal anatomy on coronary angiography. It is possible that the knife blade damaged small branches of the LAD unseen angiography, or induced temporary spasm of the main LAD. However, it remains surprising that such extensive ECG changes were seen in the face of $\supset$ a normal exercise test and perfusion scan.

In the past 7 years at Northwick Park Hospital $\stackrel{\circ}{\circ}$ there have been 7 stab wounds involving the heart; $\stackrel{\varrho}{\rightleftharpoons}$ so this type of injury is not rare. The case described here suggests that profound ECG changes seen in cardiac stab wounds may not be associated with damage to major coronary arteries. Such changes in the absence of tamponade do not necessarily indicate a poor prognosis.

\section{Acknowledgments}

We would like to thank Dr E. B. Raftery and Mr A. G. Cox for permission to publish this case and for advice in the preparation of this communication.

\section{References}

De Wet Lubbe, J.J., Janson, P.M.C. \& Barnard, P.M. (1975) Penetrating wounds of the heart and great vessels. South $\sigma$ African Medical Journal, 49, 512.

Espada, R., Whisennand, H.H., Mattox, K.L. \& Beall, 응 A.C. (1975) Surgical management of penetrating injuries $\omega$ to the coronary arteries. Surgery, 78, 755.

Rea, W.J., SugG, W.L., Wilson, L.C., WebB, W.R. \& ECKER, R.R. (1969) Coronary artery lacerations. Annals of Thoracic Surgery, 7, 518.

Tector, A.J., Reuben, C.F., Hoffman, J.F., Gelfand, E.T., $\stackrel{\mathscr{\leftrightarrow}}{+}$ KeElAN, M. \& Worman, L. (1973) Coronary artery wounds $\square$ treated with saphenous vein bypass grafts. Journal of the $\bar{O}$ Americal Medical Association, 225, 282. 